OPEN ACCESS

Edited by:

Vincent Vander Poorten,

KU Leuven, Belgium

Reviewed by:

Vincent Gregoire,

Centre Léon Bérard, France

June Corry,

GenesisCare, Australia

*Correspondence:

Sjoukje F. Oosting

s.oosting@umcg.nl

Specialty section: This article was submitted to

Head and Neck Cancer,

a section of the journal

Frontiers in Oncology

Received: 09 March 2020

Accepted: 21 April 2020

Published: 05 June 2020

Citation:

Hanemaaijer SH, Kok IC Fehrmann RSN, van der Vegt $B$,

Gietema JA, Plaat BEC, van

Vugt MATM, Vergeer MR,

Leemans CR, Langendijk JA,

Voortman J, Buter $J$ and Oosting SF (2020) Comparison of Carboplatin With 5-Fluorouracil vs. Cisplatin as

Concomitant Chemoradiotherapy for Locally Advanced Head and Neck

Squamous Cell Carcinoma.

Front. Oncol. 10:761.

doi: 10.3389/fonc.2020.00761

\section{Comparison of Carboplatin With 5-Fluorouracil vs. Cisplatin as Concomitant Chemoradiotherapy for Locally Advanced Head and Neck Squamous Cell Carcinoma}

Saskia H. Hanemaaijer ${ }^{1}$, Iris C. Kok ${ }^{2}$, Rudolf S. N. Fehrmann ${ }^{2}$, Bert van der Vegt ${ }^{3}$, Jourik A. Gietema ${ }^{2}$, Boudewijn E. C. Plaat ${ }^{1}$, Marcel A. T. M. van Vugt ${ }^{2}$, Marije R. Vergeer ${ }^{4}$, C. René Leemans ${ }^{5}$, Johannes A. Langendijk ${ }^{6}$, Jens Voortman ${ }^{7}$, Jan Buter ${ }^{7}$ and Sjoukje F. Oosting ${ }^{2 *}$

1 Department of Otorhinolaryngology, University of Groningen, University Medical Center Groningen, Groningen, Netherlands, ${ }^{2}$ Department of Medical Oncology, University of Groningen, University Medical Center Groningen, Groningen, Netherlands,

${ }^{3}$ Department of Pathology, University of Groningen, University Medical Center Groningen, Groningen, Netherlands,

${ }^{4}$ Department of Radiation Oncology, Amsterdam UMC, Vrije Universiteit Amsterdam, Amsterdam, Netherlands,

${ }^{5}$ Department of Otolaryngology/Head and Neck Surgery, Amsterdam UMC, Vrije Universiteit Amsterdam, Amsterdam,

Netherlands, ${ }^{6}$ Department of Radiotherapy, University of Groningen University Medical Center Groningen, Groningen,

Netherlands, ${ }^{7}$ Department of Medical Oncology, Amsterdam UMC, Vrije Universiteit Amsterdam, Amsterdam, Netherlands

Background: Chemoradiotherapy (CRT) including three cycles of cisplatin is considered the standard of care for locally advanced head and neck squamous cell carcinoma (LA-HNSCC). However, around one-third of the patients cannot complete cisplatin because of toxicity. Carboplatin plus 5-fluorouracil (carbo-5FU) is another accepted treatment option with a different toxicity profile. We compared tolerability and efficacy of concomitant carbo-5FU and cisplatin.

Patients and Methods: We conducted a retrospective analysis of LA-HNSCC patients treated with CRT in two Dutch cancer centers between 2007 and 2016. All patients received intensity-modulated radiotherapy. One center routinely administered carboplatin $300-350 \mathrm{mg} / \mathrm{m}^{2}$ at day 1,22 , and 43 followed by $5 F U 600 \mathrm{mg} / \mathrm{m}^{2} /$ day for $96 \mathrm{~h}$. The other center used cisplatin $100 \mathrm{mg} / \mathrm{m}^{2}$ at day 1,22 , and 43 . The primary endpoint of this study was chemotherapy completion rate. Secondary endpoints included overall survival $(\mathrm{OS})$, disease-free survival (DFS), locoregional control (LRC) and distant metastasis-free interval (DMFS), toxicity, and unplanned admissions.

Results: In the carbo-5FU cohort $(n=211), 60.2 \%$ of the patients completed chemotherapy vs. $76.7 \%(p<0.001)$ of the patients in the cisplatin cohort $(n=223)$. Univariate analysis showed a higher risk of death in the carbo-5FU cohort [hazard ratio (HR) $1.53,95 \% \mathrm{Cl}, 1.09-2.14, p=0.01$ ] with a 3 -year OS of 65.4 vs. $76.5 \%$ for cisplatin. OS was independently associated with $\mathrm{T}$ and $\mathrm{N}$ stage and $\mathrm{p} 16$ status, but not with chemotherapy regimen ( $\mathrm{HR} 1.08,95 \% \mathrm{Cl}, 0.76-1.55, p=0.65)$. Three-year DFS was $70.0 \%$ for carbo-5FU vs. $78.6 \%$ for cisplatin (HR 1.37, 95\% Cl, 0.93-2.01, $p=0.05)$. A similar outcome was observed for both LRC (HR 1.27, 95\% Cl, 0.74-2.09, 
$p=0.4)$ and DMFS (HR 1.08, 95\% Cl 0.62-1.90, $p=0.77)$. The risk of discontinuation for chemotherapy-associated toxicity was higher in the carbo-5FU cohort than in the cisplatin cohort (relative risk $=1.69$ ).

Conclusion: LA-HNSCC patients treated with concomitant carbo-5FU completed chemotherapy less frequently than patients treated with cisplatin. Treatment regimen was not an independent prognostic factor for OS.

Keywords: area under the concentration-time curve, carboplatin 5-flourouracil, chemoradiotherapy, cisplatin, comparison, head and neck squamous cell carcinoma, locally advanced head and neck squamous cell carcinoma

\section{INTRODUCTION}

Head and neck squamous cell carcinoma (HNSCC) is the seventh most common type of cancer worldwide, accounting for 600,000 new cases and 250,000 cancer deaths each year $(1,2)$. About half of the patients are diagnosed with locally advanced head and neck squamous cell carcinoma (LA-HNSCC) (3). Definitive chemoradiotherapy (CRT) is the treatment of choice in patients with unresectable LA-HNSCC. Concomitant CRT has been shown to be superior to radiotherapy alone, with an absolute survival benefit of $6.5 \%$ at 5 years, raising 5 -year overall survival (OS) from 27.2 to $33.7 \%$ (4). In addition, recent studies have indicated that higher cumulative cisplatin dose is associated with better OS $(5,6)$.

Concomitant CRT consisting of three cycles of high-dose cisplatin on day 1 , day 22 , and day 43 in combination with 70 Gray (Gy) radiotherapy in 35 fractions is considered the standard of care. However, this regimen causes significant acute and late toxicity. As a result, approximately one-third of the patients cannot complete three cisplatin cycles (7). Concomitant carboplatin plus 5-fluorouracil (carbo-5FU) has also been shown to improve OS compared to radiotherapy alone and is an accepted treatment regimen (4). However, no randomized controlled trials comparing concomitant cisplatin with concomitant carbo-5FU have been conducted. To study differences in tolerability and efficacy of both regimens, we compared all consecutive patients from one Dutch cancer center that routinely used carbo-5FU with all consecutive patients from another Dutch cancer center that routinely used highdose cisplatin.

We hypothesized that carbo-5FU is better tolerated than cisplatin and will be reflected by a higher percentage of patients completing three chemotherapy cycles, with similar efficacy. Therefore, the primary endpoint of this study was chemotherapy completion rate. Secondary endpoints included OS, disease-free survival (DFS), locoregional control (LRC) and distant metastasis-free interval, toxicity, and the number of unplanned admissions.

\section{PATIENTS AND METHODS}

\section{Patients, Study Design, and Data Collection}

We conducted a comparative retrospective cohort study of patients who received primary concomitant CRT for LAHNSCC at the University Medical Center Groningen (UMCG) or Amsterdam University Medical Center (AUMC) between July 2007 and February 2016. Inclusion criteria were histologically proven HNSCC, stage III-IVB and concomitant CRT as primary treatment. Patients with nasopharyngeal carcinoma and patients who received postoperative CRT or CRT for cancer recurrence were excluded. Chemotherapy other than carbo-5FU (UMCG) or 3-weekly cisplatin (AUMC) was an additional exclusion criterion. Both centers prospectively included all patients treated with CRT in a database; these databases were used to identify candidates for both cohorts. Information about patient demographics, comorbidity, smoking, tumor characteristics, treatment details, toxicity, and outcome were extracted from electronic patient records. Survival status of patients lost to follow-up was checked in the population register. The Dutch Medical Research Involving Human Subjects Act does not apply to data collection as part of routine clinical practice. Therefore, the local ethics committee determined that this study was exempt from the ethical approval requirement. The study was registered at ClinicalTrials.gov (Identifier: NCT02778191).

\section{Endpoints}

The primary endpoint of the study was the percentage of patients completing three chemotherapy cycles, regardless of dose reduction. Secondary endpoints included overall OS, DFS, LRC and distant metastasis-free interval, toxicity, and the number of unplanned admissions. OS was defined as the time between diagnosis and death from any cause. Patients who were alive at the last follow-up were censored. DFS was defined as the time between the last day of CRT and first evidence of relapse or death from any cause, whichever occurred first. LRC was defined as the time between the last day of CRT and first evidence of local or regional relapse. Distant metastases-free interval was defined as the time between the last day of CRT and first evidence of distant metastasis. For LRC and distant metastasisfree interval, patients who died without evidence of recurrence were censored at the date of death. Toxicity was reported according to CTCAE version 4.03. Reasons for chemotherapy discontinuation were noted.

\section{Treatment Regimens}

The carbo-5FU regimen consisted of carboplatin $300 \mathrm{mg} / \mathrm{m}^{2}$ for patients with a creatinine clearance of $80-120 \mathrm{~mL} / \mathrm{min}$ and 350 $\mathrm{mg} / \mathrm{m}^{2}$ for patients with a creatinine clearance of $>120 \mathrm{~mL} / \mathrm{min}$. Creatinine clearance was calculated using the Cockcroft-Gault formula. Carboplatin was given intravenously in $250 \mathrm{~mL}$ of dextrose $5 \%$ over $30 \mathrm{~min}$. $5 \mathrm{FU}$ was given as a continuous infusion 
at a dose of $600 \mathrm{mg} / \mathrm{m}^{2} /$ day in $2,000 \mathrm{~mL}$ of normal saline with $20 \mathrm{mmol}$ potassium for $96 \mathrm{~h}$. The whole chemotherapy cycle was given as an inpatient regimen. Chemotherapy cycles were started on day 1 , day 22, and day 43 of radiotherapy. Cisplatin $100 \mathrm{mg} / \mathrm{m}^{2}$ was given intravenously in $500 \mathrm{~mL}$ of normal saline over $3 \mathrm{~h}$ on day 1 , day 22, and day 43 of RT. Prehydration consisted of $1 \mathrm{~L}$ of normal saline plus $20 \mathrm{mmol}$ potassium and $2 \mathrm{~g}$ of magnesium sulfate over $2 \mathrm{~h}$ and posthydration consisted of $4 \mathrm{~L}$ of normal saline plus $80 \mathrm{mmol}$ of potassium and $8 \mathrm{~g}$ of magnesium sulfate over $20 \mathrm{~h}$. Dexamethasone and a 5HT3 receptor antagonist were given as antiemetics with both treatment regimens; and patients treated with cisplatin also received aprepitant. All patients received intensity modulated radiotherapy with 70 Gy in 35 daily fractions of 2 Gy on weekdays on the primary tumor and involved lymph nodes. Elective areas were treated with $54.25 \mathrm{~Gy}$ in 35 daily fractions of $1.55 \mathrm{~Gy}$.

\section{Clinical Evaluation and Follow-Up}

During treatment, patients were evaluated weekly by a radiation oncologist for locoregional toxicity. In addition, patients were evaluated by a medical oncologist before and after every chemotherapy cycle for systemic toxicity until resolution of acute side effects. Response evaluation, including clinical examination and computerized tomography (CT) or magnetic resonance imaging (MRI), was performed 8-12 weeks after CRT. In case of residual disease, salvage surgery was performed if possible. During follow-up, patients were seen by a radiation oncologist and head-and-neck surgeon every 3 months until 2 years after CRT, and thereafter every 6 months until 5 years after CRT. Imaging using CT or MRI was done in case of clinical suspicion of recurrent disease.

\section{Carboplatin Area Under the Concentration-Time Curve}

For patients who received carboplatin, the dose was calculated retrospectively as area under the curve (AUC) for each cycle. The Calvert formula was used: AUC = absolute dose (mg) carboplatin/(creatinine clearance +25$)$.

\section{Statistical Analysis}

Assuming a completion rate of $60 \%$ for cisplatin and a sampling ratio of 1 , we estimated that 353 patients had to be included to demonstrate $10 \%$ difference in completion rate in favor of carbo-5FU, with a type 1 error rate (alpha) of 5\% and a power of 0.80 .

Pearson chi-square test and Mann-Whitney $U$-test were used to compare variables between patient cohorts. Logistic regression analysis was used to analyze the association between chemotherapy completion and other variables. In the univariate analyses all patient and tumor characteristics were included. For the multivariate analyses only the factors that were significant in univariate analysis were used.

In the univariate analysis, OS, DFS, LRC, and distant metastasis-free interval were analyzed using the Kaplan-Meier method and compared with the log-rank test. Hazard ratios (HRs) were calculated with the Cox proportional hazards model. In both Kaplan Meier and Cox regression analysis, censoring was applied in case patients were lost to follow-up. In the multivariable analysis, associations between clinical and pathological parameters and OS were determined with Cox proportional hazards model using the Wald statistic. In addition, Cox regression provided an estimate of the effect of treatment on OS and DFS after the adjustment for covariates modeling the HR. In this way, we were able to investigate OS and DFS stratified for chemotherapy regimen independently of the differences in baseline characteristics.

Levene's test was used to compare variances in AUC. To determine if high carboplatin AUC during cycle one and two resulted in omission of the third cycle, we used the MannWhitney $U$ test to compare the cumulative AUC dose of cycle one plus cycle two between patients who completed three carboFU cycles and patients who completed only two cycles. Statistical tests were performed using SPSS (version 23.0 for Windows ${ }^{\circledR}$; IBM, Armonk, NY, USA).

\section{RESULTS}

\section{Demographics}

A total of 434 patients were included, 211 of whom were in the carbo-5FU cohort and 223 in the cisplatin cohort. From all consecutive patients treated at the UMCG, 30 patients received cetuximab and were therefore excluded from analysis. None of the UMCG patients was treated with cisplatin. A total of 72 patients were excluded from the AUMC cohort based on either receiving weekly cisplatin $(n=48)$ or cetuximab $(n=27)$ (Supplementary Figure 1). Of the total group of patients, $74 \%$ were men. The mean age at diagnosis was 57 years (range 29-73). The most common tumor location was the oropharynx (64\%). Patient and tumor characteristics are listed in Table 1. Detailed information on comorbidity in both study cohorts is shown in Table 2. No significant differences between patients treated with carbo-5FU and cisplatin were noted for sex, age, comorbidity, tumor site, and stage. However, there were significant differences between both study cohorts for the following variables: $\mathrm{T}$ and $\mathrm{N}$ classification, p16 status, and tobacco exposure. Patients treated with carbo-5FU had higher $\mathrm{T}$ and $\mathrm{N}$ classification and fewer $\mathrm{p} 16-$ positive oropharynx tumors and were more frequently current smokers. Because no reliable data on World Health Organization (WHO) performance score, mucositis, skin toxicity, and alcohol consumption could be derived from patient records; these variables could not be included in the analysis.

\section{Treatment Compliance}

Of the patients treated with carbo-5FU, $60.2 \%$ completed three chemotherapy cycles vs. $76.7 \%$ of the patients treated with cisplatin $(p<0.001)$. Only $4.4 \%$ of the patients in the carbo$5 \mathrm{FU}$ cohort and $6.7 \%$ of the patients in the cisplatin cohort had chemotherapy dose reductions $(p=0.3)$. The reasons for discontinuation of chemotherapy are listed in Table 3.

\section{Outcome}

The median follow-up was 27 months (range 1-109) in the carbo5FU cohort and 35 months (range 1-111) in the cisplatin cohort. In the carbo-5FU cohort, 79 patients had died (37.4\%), compared 
TABLE 1 | Baseline patient characteristics.

\begin{tabular}{|c|c|c|c|}
\hline Characteristics & $\begin{array}{c}\text { Carbo-5FU } \\
(n=211)\end{array}$ & $\begin{array}{l}\text { Cisplatin } \\
(n=233)\end{array}$ & $p$ \\
\hline Age, years & & & 0.605 \\
\hline [Median (IQR)] & [58 (53-63)] & [58 (52-63)] & \\
\hline Sex & & & 0.186 \\
\hline Male & 149 (70.6) & $170(76.2)$ & \\
\hline Female & $62(29.4)$ & $53(23.8)$ & \\
\hline Comorbidity & & & 0.902 \\
\hline Yes & 75 (35.3) & $78(35.0)$ & \\
\hline No & $136(64.5)$ & $145(65.0)$ & \\
\hline Tumor site & & & 0.869 \\
\hline Oral cavity & $14(6.6)$ & $25(11.2)$ & \\
\hline Hypopharynx & $30(14.2)$ & $38(17.0)$ & \\
\hline Larynx & $24(11.4)$ & $26(11.7)$ & \\
\hline Oropharynx & $143(67.8)$ & $134(60.1)$ & \\
\hline $\mathrm{T}$ classification & & & 0.002 \\
\hline T1 & $18(8.5)$ & $23(10.3)$ & \\
\hline T2 & 39 (18.5) & $49(22.0)$ & \\
\hline T3 & $55(26.1)$ & $88(39.5)$ & \\
\hline T4 & 99 (46.9) & $63(28.3)$ & \\
\hline $\mathrm{N}$ classification & & & $<0.001$ \\
\hline NO & $14(6.6)$ & $40(17.9)$ & \\
\hline N1 & $17(8.1)$ & $42(18.8)$ & \\
\hline N2 & $171(81.0)$ & $135(60.5)$ & \\
\hline N3 & $9(4.3)$ & $6(2.7)$ & \\
\hline AJCC stage & & & 0.650 \\
\hline III & 27 (12.8) & 45 (20.2) & \\
\hline Iva & $162(76.8)$ & $158(70.9)$ & \\
\hline $\mathrm{IVb}$ & $22(10.4)$ & $20(9.0)$ & \\
\hline p16 (in oropharynx tumors) & & & $<0.001$ \\
\hline Positive & $65(45.4)$ & $85(63.4)$ & \\
\hline Negative & $73(51.0)$ & $39(29.1)$ & \\
\hline Unknown & $5(3.5)$ & $10(7.5)$ & \\
\hline Tobacco exposure & & & 0.565 \\
\hline Current smoker & $138(65.4)$ & 97 (43.5) & \\
\hline Former smoker & $53(25.1)$ & $82(36.8)$ & \\
\hline Never smoked & $20(9.5)$ & 44 (19.7) & \\
\hline Pack-years & & & 0.262 \\
\hline Zero & $20(9.5)$ & $44(19.7)$ & \\
\hline$<10$ & $3(1.4)$ & $8(3.6)$ & \\
\hline$>10$ & $167(79.1)$ & $143(64.1)$ & \\
\hline Missing & 21 (10.0) & 28 (12.6) & \\
\hline
\end{tabular}

Data are presented as No. (\%) unless otherwise indicated. The Mann-Whitney U-test was used. AJCC, American Joint Committee on Cancer (7th edition); IQR, interquartile range. Tobacco exposure is defined as current/former smoker.

to $61(27.4 \%)$ in the cisplatin cohort (Table 4$)$. The cause of death was tumor-related in 53 patients $(25.1 \%)$ in the carbo$5 F U$ cohort and in 45 patients $(20.2 \%)$ in the cisplatin cohort. Treatment-related deaths were observed in 3 patients (1.4\%) in the carbo-5FU cohort (heart failure, sepsis, and sudden death) and in 2 patients $(0.9 \%)$ in the cisplatin cohort (pneumonia and sudden death).
TABLE 2 | Baseline comorbidity in the carbo-5FU and in the cisplatin cohort.

\begin{tabular}{|c|c|c|}
\hline Characteristics & $\begin{array}{c}\text { Carbo-5FU } \\
(n=211)\end{array}$ & $\begin{array}{l}\text { Cisplatin } \\
(n=233)\end{array}$ \\
\hline
\end{tabular}

Comorbidity

Myocardial infarction

Peripheral vascular disease

Diabetes mellitus

Pulmonary embolism

COPD

Cerebrovasculair accident/TIA

Other malignancy ${ }^{\mathrm{C}}$

18
14
11
2
11
11
17

$\begin{array}{cc} & 0.902^{\mathrm{b}} \\ 19 & 1.00 \\ 4 & 0.15 \\ 16 & 0.432 \\ 2 & 1.00 \\ 9 & 0.217 \\ 9 & 0.649 \\ 30 & 0.089\end{array}$

COPD, chronic obstructive pulmonary disease; TIA, transient ischemic attack.

a Pearson chi square test was used unless otherwise indicated.

${ }^{b}$ Mann-Whitney U-test was used.

${ }^{c}$ Malignancy in medical history.

TABLE 3 | Reasons for discontinuation of chemotherapy.

\begin{tabular}{lccc}
\hline Reasons $^{\text {a }}$ & $\begin{array}{c}\text { Carbo-5FU } \\
(\boldsymbol{n}=\mathbf{2 1 1})\end{array}$ & $\begin{array}{c}\text { Cisplatin } \\
(\boldsymbol{n}=\mathbf{2 3 3})\end{array}$ & $\boldsymbol{p}$ \\
\hline Thrombocytopenia & $52(24.6)$ & $1(0.4)$ & $<0.001$ \\
Acute kidney injury & $2(0.9)$ & $21(9.4)$ & $<0.001$ \\
Leukocytopenia & $19(9.0)$ & $6(2.6)$ & 0.005 \\
Ototoxicity & $0(0)$ & $8(3.5)$ & 0.005 \\
Emesis & $0(0)$ & $5(2.2)$ & 0.029 \\
Performance & $4(1.9)$ & $1(0.4)$ & 0.158 \\
Patient request & $5(2.3)$ & $2(0.9)$ & 0.223 \\
Allergic reaction & $2(0.9)$ & $0(0)$ & 0.145 \\
Anemia & $4(1.9)$ & $1(0.4)$ & 0.158 \\
Other & $11(1.4)$ & $8(3.1)$ & 0.408 \\
\hline
\end{tabular}

Data presented as No. (\%) unless otherwise indicated. The Mann-Whitney U-test was used.

${ }^{a}$ One or more reasons could be reported per patient.

Median OS was 65 months in the carbo-5FU cohort and not reached in the cisplatin cohort. The risk of death was higher in the carbo-5FU cohort (HR 1.53, 95\% CI, 1.09-2.14, $p=0.01)$. 1-year and 3-year OS was 86.7 and $65.5 \%$ for carbo5FU and $89.9 \%$ and $76.6 \%$ for cisplatin (Figure 1A). Univariate analysis demonstrated that lower T-classification, lower $\mathrm{N}$ classification, p16 positivity (in oropharyngeal tumors), nonsmoking, cisplatin chemotherapy, and the absence of a second primary tumor were associated with better OS (Table 5). Sex, age, and tumor site were not associated with OS. Multivariate analysis showed that lower $\mathrm{T}$ classification and lower $\mathrm{N}$ classification, p16 positivity (in oropharyngeal tumors), and the absence of second primaries were independently associated with better OS (Table 5). Chemotherapy regimen (HR 1.08, 95\% CI, 0.761.55) and smoking (HR 1.20, 95\% CI, 0.91-1.60) were not independently associated with OS. Kaplan-Meier curves for OS of both study cohorts and adjusted OS curves according to the 
Cox proportional hazards model (labeled with an asterisk) are shown in Figure 1A.

Median DFS was not reached in either cohort. One-year and 3-year DFS were 77.5 and $70.0 \%$ for carbo-5FU compared to 84.5 and $78.6 \%$ for cisplatin (HR 1.37, 95\% CI, $0.93-$ 2.01, $p=0.05$, Figure 1B). Kaplan-Meier curves for DFS of both study cohorts and adjusted DFS curves according to the Cox proportional hazard model (labeled with an asterisk) are shown in Figure 1B. Similar outcome was observed for both LRC (HR 1.27, 95\% CI, 0.74- 2.09, $p=0.4$ ) and distant metastasis-free interval (HR 1.08, 95\% CI, 0.62-1.90, $p=0.77$ ) as presented in Figure 2.

TABLE 4 | Disease and survival status of patients at the time of analysis.

\begin{tabular}{lccc}
\hline & $\begin{array}{c}\text { Carbo-5FU } \\
\boldsymbol{n}=\mathbf{2 1 1}\end{array}$ & $\begin{array}{c}\text { Cisplatin } \\
\boldsymbol{n}=\mathbf{2 3 3}\end{array}$ & $\boldsymbol{p}^{\mathbf{a}}$ \\
\hline Survival status & & & 0.03 \\
NED & $128(60.7)$ & $155(69.5)$ & \\
AWD & $4(1.9)$ & $7(3.1)$ & \\
DOD & $52(24.6)$ & $44(19.7)$ & \\
DOC & $26(12.3)$ & $17(7.6)$ & \\
Missing death & $1(0.5)$ & $0(0)$ & \\
Event & & & \\
Residual disease & $13(6.2)$ & $5(2.2)$ & 0.041 \\
Local regional recurrence & $28(13.3)$ & $30(13.5)$ & 0.611 \\
Distant metastasis & $24(11.3)$ & $25(11.2)$ & 0.957 \\
Local regional + distant metastasis & $49(23.2)$ & $47(21.1)$ & 0.590 \\
\end{tabular}

a Mann-Whitney U-test was used. NED, no evidence of disease; AWD, alive with disease; $D O D$, died of disease; DOC, died of other cause.
In a combined analysis of the treatment groups, a trend toward better OS in patients who completed three cycles of chemotherapy compared to the patients who completed one or two cycles of chemotherapy was observed (5-year OS 61.6 vs. $53.7 \%$, HR 1.32, 95\% CI, 0.92-1.89, $p=0.11$ ); see Figure 3.

\section{Treatment-Related Toxicity}

Thrombocytopenia was the main reason for discontinuation in the carbo-5FU cohort. Acute kidney injury followed by ototoxicity was the most common reason for not completing three cycles of cisplatin. The risk of discontinuation for chemotherapy-associated toxicity was higher in the carbo-5FU cohort than in the cisplatin cohort (relative risk $=1.69$ ). There was no difference in radiotherapy completion rate between study cohorts: $97.7 \%$ for the carbo-5FU cohort vs. $95.5 \%$ for the cisplatin cohort $(p=0.3)$. In multivariate analysis, chemotherapy completion was negatively associated with carbo-5FU, female sex, and comorbidity. No association between chemotherapy completion rate and $\mathrm{T}$ classification, $\mathrm{N}$ classification, tumor location, age, smoking status, or p16 status was found.

Grade 3 or 4 anemia was seen only in the carbo-5FU cohort and occurred in $4.7 \%$ of the patients. Grade 3 or 4 thrombocytopenia was observed more frequently in the carbo$5 \mathrm{FU}$ cohort compared to the cisplatin cohort (13.7 vs. $1.8 \%, p$ $<0.001)$. Neutropenic fever was uncommon in both cohorts, whereas leukopenia was frequently reported in both groups: in $15.6 \%$ of the patients in the carbo-5FU cohort and in $21.1 \%$ in the cisplatin cohort $(p=0.430)$. Acute kidney injury grade $3-4$ was observed only in the cisplatin cohort, in 13 patients (6 vs. $0 \%, p<0.001)$. At $6-12$ weeks after treatment, 7 of these patients (3\%) still had treatment-related kidney injury.

Almost $80 \%$ of the patients in the carbo-5FU cohort received carboplatin, with an AUC between 4 and 6 (Figure 4). The
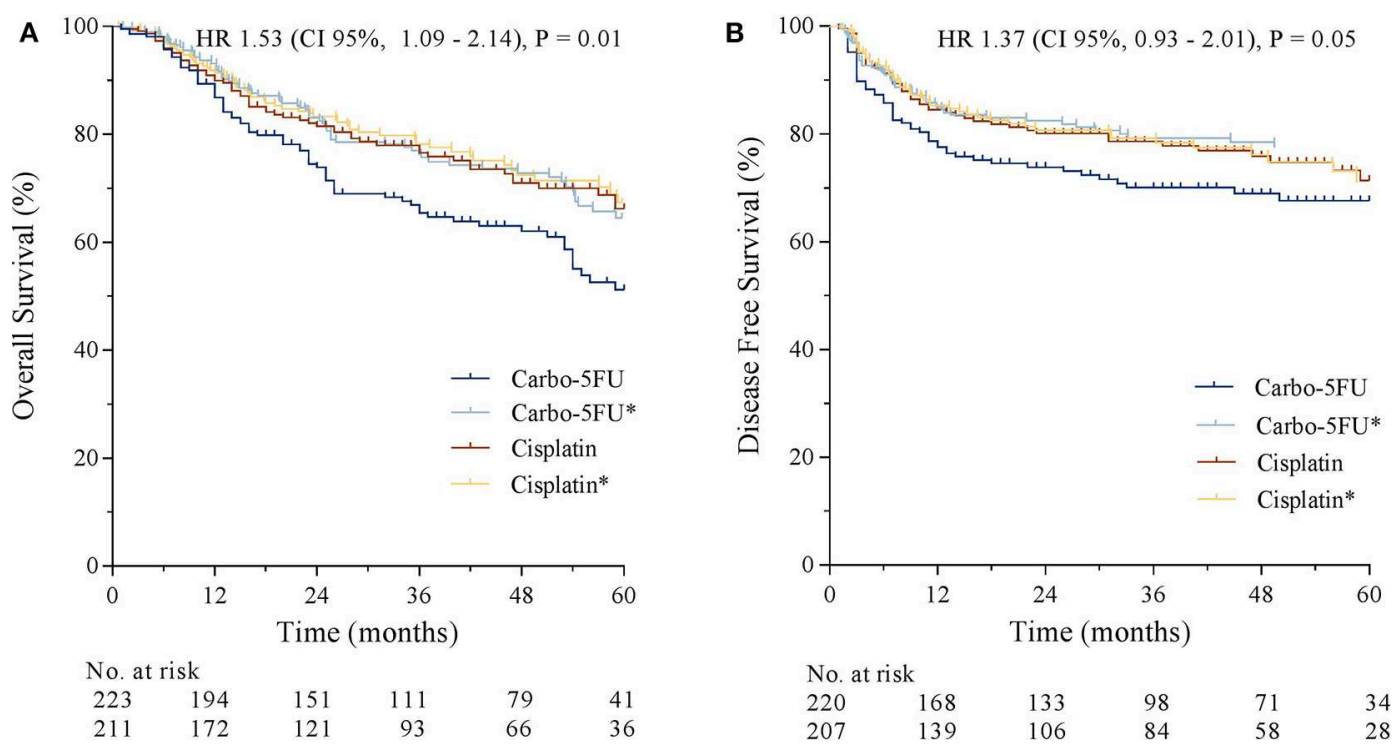

FIGURE 1 | (A) Overall survival stratified by chemotherapy regimen. (B) Disease-free survival stratified by chemotherapy regimen. The adjusted overall survival estimation for both cohorts is labeled with an asterisk. 
mean AUC of the cycles received was $<4.00$ in 36 patients $(17 \%)$ and $>7.00$ in 8 patients (3.8\%). Four of these 8 patients could not continue therapy because of treatment-related toxicity. Patients who completed three carbo-5FU cycles received a similar cumulative carboplatin dose during the first two cycles [median

TABLE 5 | Significant Variables Univariate Analysis and Multivariate Analysis for Overall Survival.

\begin{tabular}{|c|c|c|c|}
\hline Variable & HR & $95 \% \mathrm{Cl}$ & $p$ \\
\hline \multicolumn{4}{|l|}{ Univariate analysis } \\
\hline T-classification T1-T2 & 1 (ref) & & \\
\hline Т3-T4 & 4.04 & $2.400-6.803$ & $<0.001$ \\
\hline N-classification NO-N2a & 1 (ref) & & \\
\hline N2bc-N3 & 1.83 & $1.244-2.700$ & 0.002 \\
\hline p16 (in oropharynx tumors) & 0.25 & $0.153-0.411$ & $<0.001$ \\
\hline Second primary ${ }^{a}$ & 2.38 & $1.252-4.541$ & 0.008 \\
\hline Treatment regimen & 1.53 & $1.094-2.136$ & 0.013 \\
\hline Tobacco exposure & 1.30 & $1.779-1.669$ & 0.041 \\
\hline \multicolumn{4}{|l|}{ Multivariate analysis } \\
\hline T-classification T1-T2 & 1 (ref) & & \\
\hline Т3-Т4 & 3.42 & $1.984-5.882$ & $<0.001$ \\
\hline N-classification NO-N2a & 1 (ref) & & \\
\hline N2bc-N3 & 2.23 & $1.464-3.380$ & $<0.001$ \\
\hline p16 (in oropharynx tumors) & 0.35 & $0.211-0.591$ & $<0.001$ \\
\hline Second primary ${ }^{a}$ & 2.98 & $1.464-5.851$ & 0.001 \\
\hline Treatment regimen & 1.19 & $0.835-1.709$ & 0.331 \\
\hline Tobacco exposure & 1.15 & $0.859-1.538$ & 0.349 \\
\hline
\end{tabular}

Cox regression was used. (ref) denotes for reference variable. Tobacco exposure was defined as current/former smoker or non-smoker.

a Second primary in the Head and Neck region at time of initial diagnosis.
8.78, interquartile range (IQR) 7.90-9.75, min-max 3.28-12.40] compared to patients that completed two carbo-5FU cycles (median 8.71, IQR 7.84-9.74, min-max 4.52-13.83, $p=0.85$ ). No association was found between carboplatin dose and grade 3 or 4 thrombocytopenia, leukocytopenia, or anemia.

\section{Extra Hospital Admissions}

Patients treated with cisplatin were more likely to have extra hospital admissions (57.4 vs. $18.5 \%, p<0.001$ ) during CRT, mainly due to dehydration/acute kidney injury. The median duration of unplanned admissions was 4 days in the cisplatin

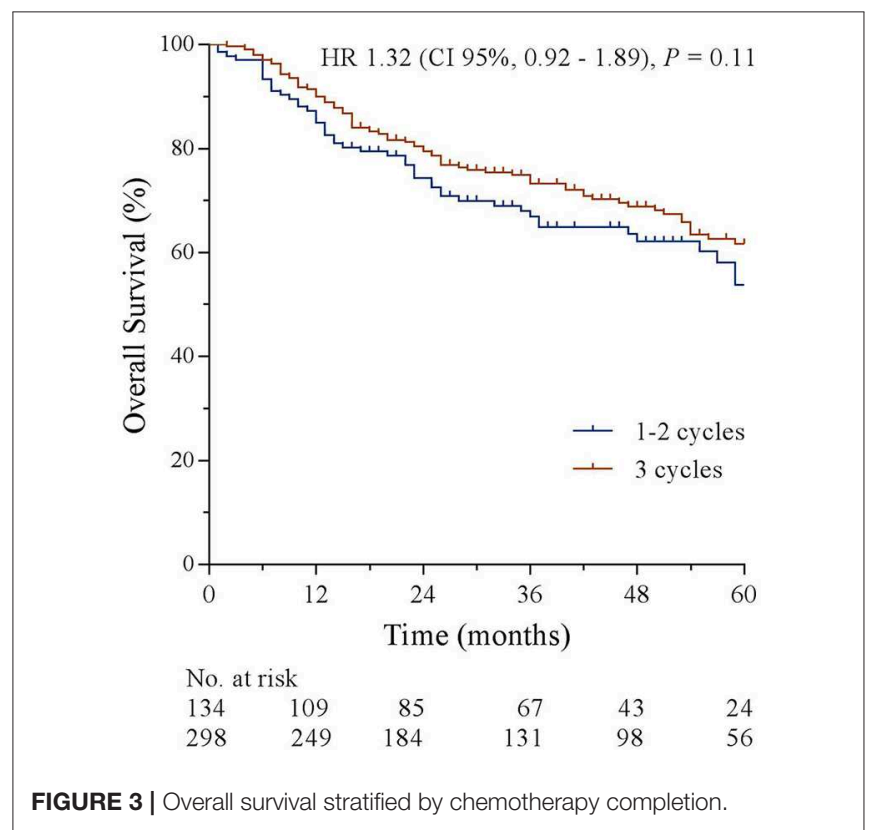

FIGURE 3 | Overall survival stratified by chemotherapy completion.
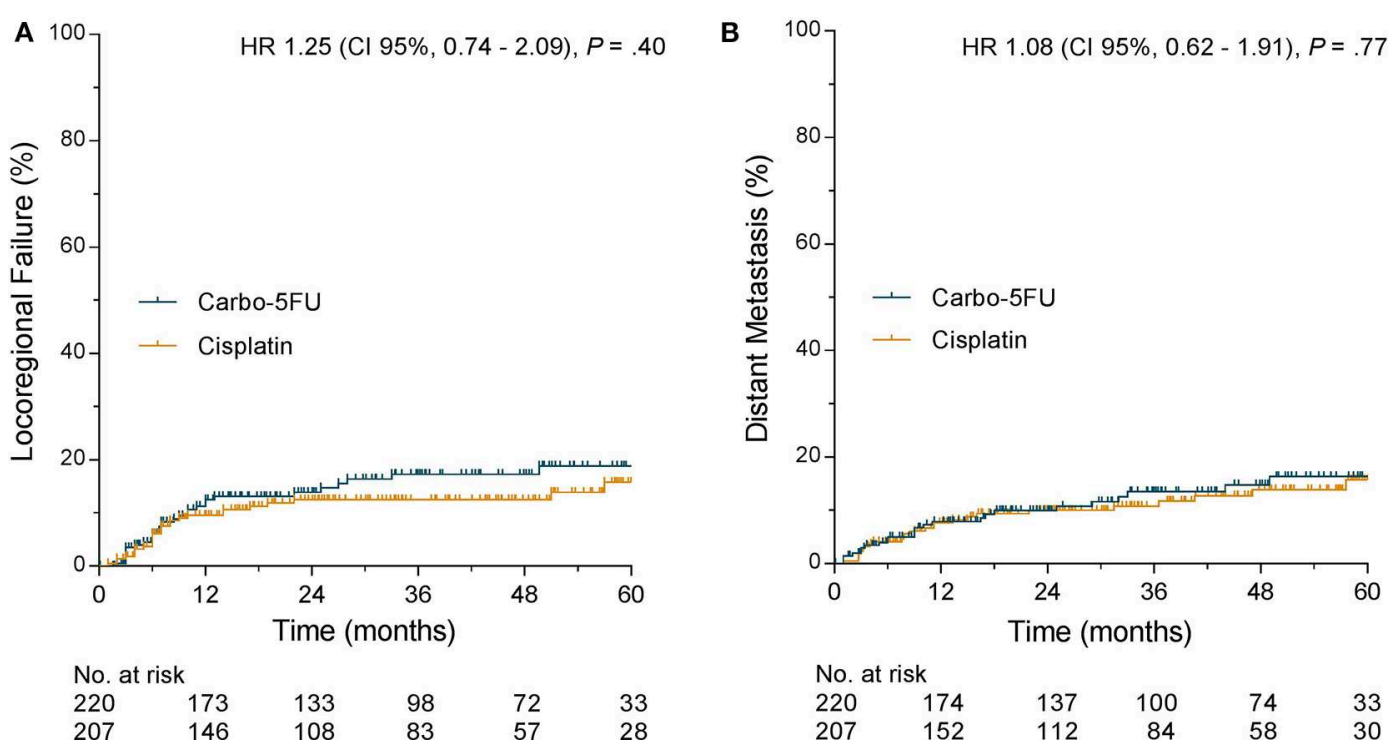

FIGURE 2 | (A) Locoregional failure stratified by chemotherapy regimen. (B) Distant metastasis-free interval stratified by chemotherapy regimen. 


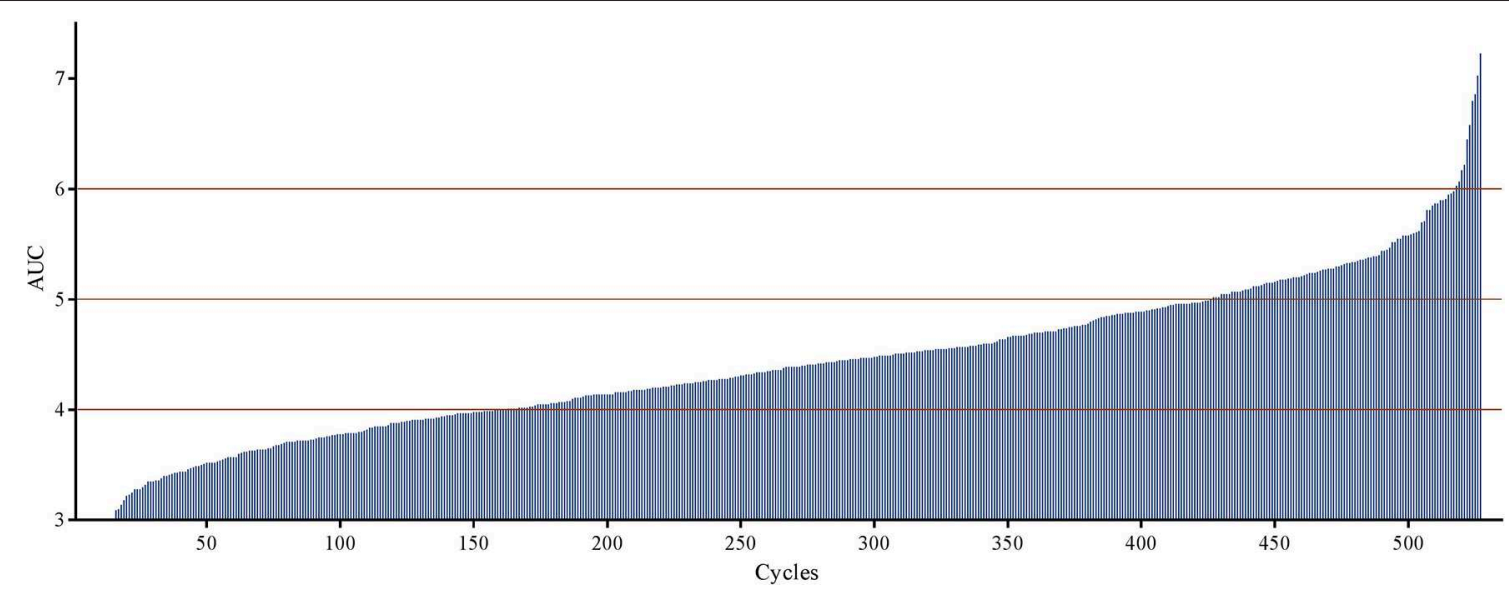

FIGURE 4 | Distribution of carboplatin AUC. Each vertical bar on the horizontal axis represents a chemotherapy cycle.

TABLE 6 | Reasons Extra Hospital Admission.

\begin{tabular}{lccc}
\hline & $\begin{array}{c}\text { Carbo-5FU } \\
\boldsymbol{n}=\mathbf{2 1 1}\end{array}$ & $\begin{array}{c}\text { Cisplatin } \\
\boldsymbol{n}=\mathbf{2 3 3}\end{array}$ & $\boldsymbol{p}^{\mathbf{a}}$ \\
\hline Characteristics & & & $>0.001$ \\
Extra admission & & & \\
One & $39(18.5)$ & $128(57.4)$ & 0.171 \\
Two & $5(2.4)$ & $53(23.8)$ & \\
Duration of admission & & & \\
[median(IQR)] & {$[5(4-9)]$} & {$[4(3-7)]$} & \\
Reason for admission & & $79(35.4)$ & \\
Decreased renal function & $0(0)$ & $44(19.7)$ & \\
Nausea and vomiting & $6(2.8)$ & $14(6.27)$ & \\
Fever & $22(10.4)$ & $15(6.7)$ & \\
Dehydration & $1(0.5)$ & $8(3.1)$ & \\
Diminished performance & $3(1.4)$ & $7(2.7)$ & \\
Infection & $3(1.4)$ & $13(5.8)$ & \\
Tumor related & $3(1.4)$ & $6(2.8)$ & \\
Other & &
\end{tabular}

${ }^{a}$ Mann-Whitney $U$ test was used. IQR, inter quartile range.

cohort, with an IQR of 4-9 (range 1-28) and 5 days in the carbo-5FU cohort (IQR 3-7, range 1-60). Reasons for each extra hospital admission are reported in Table 6.

\section{DISCUSSION}

The purpose of this study was to compare tolerability and efficacy of concomitant CRT with carbo-5FU and concomitant CRT with cisplatin in locally advanced HNSCC. We hypothesized that carbo-5FU is better tolerated than cisplatin and therefore results in a higher percentage of patients completing three chemotherapy cycles with similar efficacy. However, the results showed that patients in the carbo-5FU cohort were less likely to complete three cycles of chemotherapy than patients in the cisplatin cohort.
The observed completion rate of $76.8 \%$ in the cisplatin cohort was higher than expected, and even higher than in two randomized controlled trials, where one would expect selection of fitter patients compared to our real-life data $(7,8)$. A factor that might have influenced cisplatin completion rate is the proportion of p16-positive oropharyngeal cancer patients, which is rising over time. Since p16-positive oropharyngeal cancer usually occurs in younger patients with a better performance status, fewer smoking pack-years, and less comorbidity, it is likely that these patients are more often capable of completing three cycles. However, we found no association between p16 status and chemotherapy completion. Another factor could be the rigorous supportive care given by specialized oncology nurse practitioners. Vigorous hydration and frequent extra admissions could possibly have led to higher chemotherapy compliance and therefore should be taken into consideration for generalizability of the results.

In a trial comparing CRT with carbo-5FU to RT alone for stage III-IVB HNSCC, 65\% of the patients completed three cycles of chemotherapy (9). This is comparable to the completion rate $(60.2 \%)$ that we found. In our study, the reasons for not completing chemotherapy differed between cohorts, with more thrombocytopenia and leucopenia reported in the carbo-5FU cohort and more kidney injury and ototoxicity in the cisplatin cohort. This corresponds to the known toxicity profiles of both platinum agents (9-11). In the carbo-5FU cohort, $24.6 \%$ of the patients could not complete chemotherapy because of thrombocytopenia. If the platelet count was $<100 \times 10^{9} / \mathrm{L}$ on day 22 or day 43 , the cycle was postponed. This usually resulted in omission of the third cycle because it could not be given within the radiotherapy interval.

Although OS was significantly better in the cisplatin cohort, the well-known prognostic factors were all advantageous for this cohort. After correcting for $\mathrm{T}$ stage, N stage, p16 status, and second primaries in multivariate analysis, we found that treatment regimen was not an independent prognostic factor for OS (12). Similar DFS, LRC, and distant metastasis-free interval were observed in both univariate and multivariate analysis. 
Our results are similar to those of two previous comparative studies that demonstrated no significant difference in outcome between patients who received cisplatin and those who received carbo-5FU $(13,14)$. However, these single-center retrospective studies were restricted by statistical power and potential selection bias $(13,14)$.

Furthermore, we investigated whether treatment delay due to thrombocytopenia could have been caused by a relatively high carboplatin dose because dosing was based on body surface area rather than on creatinine clearance. However, we did not find an association between carboplatin AUC and grade 3 or grade 4 thrombocytopenia. Neither did we find a difference between cumulative carboplatin AUC of the first two cycles between patients who completed three cycles and those who completed two cycles.

Another interesting strategy that could reduce acute toxicity of concomitant CRT and further increase compliance comes from the Radiation Therapy Oncology Group 0129 trial. This study demonstrated similar efficacy of two cycles of high-dose cisplatin plus accelerated fractionation radiotherapy compared to three cycles of cisplatin plus standard fractionation (15). In the experimental arm, $87.8 \%$ of the patients completed two cycles of cisplatin whereas in the standard arm $69.0 \%$ completed three cycles. However, no difference in grade 3 or higher toxicity was found.

A limitation of our study is a difference in baseline characteristics between the cohorts due to the non-randomized retrospective design. Although this difference does not affect interpretation of chemotherapy completion rate, which was associated with treatment regimen, sex, and comorbidity, it does complicate interpretation of efficacy endpoints. However, a future randomized controlled trial investigating carbo-5FU vs. cisplatin is unlikely because the treatment focus has shifted toward immunotherapy and treatment de-escalation for lowrisk p16-positive oropharyngeal cancer. Therefore, comparing

\section{REFERENCES}

1. Ferlay J, Soerjomataram I, Dikshit R, Eser S, Mathers C, Rebelo M, et al. Cancer incidence and mortality worldwide: sources, methods and major patterns in GLOBOCAN 2012. Int J Cancer. (2015) 136:E35986. doi: $10.1002 /$ ijc. 29210

2. Jemal A, Siegel R, Ward E, Hao Y, Xu J, Thun MJ. Cancer statistics, 2008. CA Cancer J Clin. (2008) 58:71-96. doi: 10.3322/CA.2007.0010

3. Argiris A, Karamouzis MV, Raben D, Ferris RL. Head and neck cancer. Lancet. (2008) 371:1695-709. doi: 10.1016/S0140-6736(08) 60728-X

4. Pignon JP, Maître le A, Maillard E, Bourhis J, MACH-NC Collaborative Group. Meta-analysis of chemotherapy in head and neck cancer (MACH-NC): An update on 93 randomised trials and 17,346 patients. Radiother Oncol. (2009). 92:4-14. doi: 10.1016/j.radonc.2009. 04.014

5. Strojan P, Vermorken JB, Beitler JJ, Saba NF, Haigentz M Jr, Bossi P, et al. A. Cumulative cisplatin dose in concurrent chemoradiotherapy for head and neck cancer: A systematic review. Head Neck. (2016) 38:E21518. doi: $10.1002 /$ hed 24026

6. Spreafico A, Huang SH, Xu W, Granata R, Liu CS, Waldron JN, et al. Impact of cisplatin dose intensity on human papillomavirus-related and -unrelated patient cohorts from two tertiary care centers in the same country and geographic region with different institutional practice provides the best level of evidence attainable in current clinical practice. Another limitation of this study is that we were not able to retrieve reliable information on performance status, mucositis, skin toxicity, and alcohol consumption because this was not registered systematically.

To our knowledge, this is the first well-powered retrospective cohort study in which carbo-5FU and cisplatin as concomitant CRT for locally advanced HNSCC are compared with regard to chemotherapy completion. A lower chemotherapy completion rate was found for patients treated with carbo-5FU compared to patients treated with cisplatin. However, chemotherapy regimen was not independently associated with OS. We therefore believe that both chemotherapy regimens are viable treatment options for concomitant CRT in patients with locally advanced HNSCC.

\section{DATA AVAILABILITY STATEMENT}

The datasets generated for this study are available on request to the corresponding author.

\section{AUTHOR CONTRIBUTIONS}

SH, IK, JL, JB, and SO: analysis and interpretation of data. JG, $\mathrm{BP}, \mathrm{MVe}, \mathrm{CL}, \mathrm{JL}, \mathrm{JV}, \mathrm{JB}$, and SO: provision of study materials or patients. All authors are accountable for all aspects of the work, gave final approval of the manuscript, and contributed to drafting the manuscript or revising it critically.

\section{SUPPLEMENTARY MATERIAL}

The Supplementary Material for this article can be found online at: https://www.frontiersin.org/articles/10.3389/fonc. 2020.00761/full\#supplementary-material

locally advanced head and neck squamous cell carcinoma. Eur J Cancer. (2016) 67:174-82. doi: 10.1016/j.ejca.2016.08.013

7. Forastiere AA, Goepfert H, Maor M, Pajak TF, Weber R, Morrison $\mathrm{W}$, et al. Concurrent chemotherapy and radiotherapy for organ preservation in advanced laryngeal cancer. $N$ Engl J Med. (2003) 349:2091-8. doi: 10.1056/NEJMoa031317

8. Ang KK, Harris J, Wheeler R, Weber R, Rosenthal DI, Nguyen-Tan PF, et al. Human papillomavirus and survival of patients with oropharyngeal cancer. $N$ Engl J Med. (2010) 363:24-35. doi: 10.1056/NEJMoa0912217

9. Calais G, Alfonsi M, Bardet E, Sire E, Germain T, Bergerot P, et al. Randomized trial of radiation therapy versus concomitant chemotherapy and radiation therapy for advanced-stage oropharynx Carcinoma. J Natl Cancer Inst. (1990) 91:2081-6. doi: 10.1093/jnci/91.24.2081

10. Wilkins AC, Rosenfelder N, Schick U, Gupta A, Thway K, Nutting CM, et al. Equivalence of cisplatin and carboplatin-based chemoradiation for locally advanced squamous cell carcinoma of the head and neck: a matchedpair analysis. Oral Oncol. (2013) 49:615-9. doi: 10.1016/j.oraloncology.2013. 02.004

11. Fountzilas G, Ciuleanu E, Dafni U, Plataniotis G, Kalogera-Fountzila A, Samantas E, et al. Concomitant radiochemotherapy vs radiotherapy alone in patients with head and neck cancer: a hellenic cooperative oncology group phase III study. Med Oncol. (2004) 21:95-107. doi: 10.1385/MO:21:2:095 
12. Brandwein-Gensler M, Smith RV. Prognostic indicators in head and neck oncology including the New 7th edition of the AJCC staging system. Head Neck Pathol. (2010) 4:53-61. doi: 10.1007/s12105-010-0161-y

13. Barkati M, Fortin B, Soulières D, Clavel S, Despres P, Charpentier D, et al. Concurrent chemoradiation with carboplatin-5-fluorouracil versus cisplatin in locally advanced cropharyngeal cancers: is more always better? Int J Radiat Oncol Biol Phys. (2010) 76:410-6. doi: 10.1016/j.ijrobp.2009. 02.034

14. Saphiro L, Sherman E, Riaz N, Setton J, Koutcher L, Zhang Z, et al. Efficacy of concurrent cetuximab vs 5-flourouracil/carboplatin or high-dose cisplatin with intensity modulated radiation therapie (IMRT) for locallyadvanced head and neck cancer (LAHNSCC). Oral Oncol. (2014) 50:94755. doi: 10.1016/j.oraloncology.2014.07.001

15. Nguyen-Tan PF, Zhang Q, Ang KK, Weber RS, Rosenthal DI, Soulieres D, et al. Randomized phase III trial to test accelerated versus standard fractionation in combination with concurrent cisplatin for head and neck carcinomas in the radiation therapy oncology group 0129 trial: long-term report of efficacy and toxicity. J Clin Oncol. (2014) 32:3858-67. doi: 10.1200/JCO.2014. 55.3925

Conflict of Interest: BV has a consulting/advisory role for Philips Computational Pathology and is a scientific advisory board member for
Visiopharm. JG has received research grants from Abbvie, Roche, and Siemens. MVe has a consulting/advisory role for Repare Therapeutics and has received travel/accommodation support from Repare Therapeutics. JB has a consulting/advisory role for MSD, Merck BV, and BMS, and has received travel/accommodation support from MSD. Furthermore, JB has participated in a speakers' bureau for BMS, and AstraZeneca. BP has a consulting/advisory role for Olympus Medical Systems and has received grants and travel/accommodation support from Olympus Medical Systems. JL has a consulting/advisory role for JBA. SO has received grants from Celldex.

The remaining authors declare that the research was conducted in the absence of any commercial or financial relationships that could be construed as a potential conflict of interest.

Copyright (C) 2020 Hanemaaijer, Kok, Fehrmann, van der Vegt, Gietema, Plaat, van Vugt, Vergeer, Leemans, Langendijk, Voortman, Buter and Oosting. This is an open-access article distributed under the terms of the Creative Commons Attribution License (CC BY). The use, distribution or reproduction in other forums is permitted, provided the original author(s) and the copyright owner(s) are credited and that the original publication in this journal is cited, in accordance with accepted academic practice. No use, distribution or reproduction is permitted which does not comply with these terms. 